\title{
Correlation of Clinico-Histopathological Findings in Leprosy Patients at Tertiary Care Hospital, Bhilai, CG
}

\section{Bhuvaneshwari Dewangan}

Assistant Professor, Department of Dermatology, Shri Shankaracharya Institute of Medical Sciences, Bhilai

\begin{abstract}
Background: Leprosy continues to be public health problem in India. In India, Chhattisgarh state is having the highest prevalence rate of 2.33 per 10000 populations presently. Leprosy expresses itself in different clinics histopathological forms depending on the immune system the host. Objective: To find the strength of the relationship between clinically diagnosed leprosy patients with histopathological findings.
\end{abstract}

Materials \& Methods: This was a prospective study was carried out on 42 new cases of leprosy attending the outpatient department of dermatology and venereology of J. L. N. Hospital and Research Centre Bhilai Steel Plant Bhilai from March 2009 to December 2010 \& all the patients were included after taking informed consent. All the cases were subjected detailed history and through clinical examination \& histopathological examination of skin tissue as per the structured pro forma.

Results: The Male female ratio was 1:1. Majority of the patients were belonged to the age group between 41 to 60 years. Overall positive correlation found between Clinico histopathological was 0.69 . Majority of the patients were found in the borderline tuberculoid leprosy in Clinically \& Histopathological findings. The overall agreement was good between clinical spectrum and histopathological findings.

Conclusion: In some early cases, clinical signs and symptoms may precede the presently known characteristic tissue changes or vice versa. If a biopsy is taken at an early stage, there is likely to be discordance between the clinical and histopathological observations.
Keywords: Leprosy, Clinical Examination, Histopathological Examination, Strength of relationship.

\section{INTRODUCTION}

Leprosy continues to be a public health problem in India. ${ }^{(1)}$ It is also known as Hansen's disease. ${ }^{(2)}$ Globally, pockets of high endemicity still remain in some areas of Angola, Brazil, Central African Republic, Democratic Republic of Congo, India, Madagascar, Mozambique, Nepal and the United Republic of Tanzania. (3) These countries remain highly committed to eliminating the disease and continue to intensify their leprosy control activities. ${ }^{(4)}$

In India, Chhattisgarh state is having the highest prevalence rate of 2.33 per 10,000 populations presently. (5) WHO adopted its 'Enhanced Global Strategy for further reducing the disease burden due to leprosy (Plan period: 2011 - 2015)' in 2009. (6)

India has launched MDT in 1982. After introduction of MDT, spectacular success has been achieved in reducing the disease burden in our country. The national prevalence rate which stood at estimated 57.6 per 10,000 in 1981 ; has come down to 0.72 per 10,000 by March 2009. Leprosy elimination at the National level was achieved on December 31, 2005 with the recorded prevalence as 0.95 per 10,000 populations. ${ }^{(7)}$

32 states/ UT's have achieved the status of leprosy elimination. Only 3 states/ Its viz. Bihar, Chhattisgarh, Dadra \& Nagar Haveli are yet to achieve the elimination 
level (PR between 1 and 2.5 per 10,000 populations by the end of March 31, 2009). ${ }^{(7)}$

\section{Objective:}

To study the new cases of leprosy attending our OPD under following headings:

1) The Clinical \& Histopathological Examination.

2) To find the Strength of relationship between clinical \& histopathological.

\section{MATERIALS \& METHOD}

This is a prospective study was carried out on 42 new cases of leprosy attending the outpatient department of dermatology and venereology of J. L. N. Hospital and Research Centre Bhilai Steel Plant Bhilai from March 2009 to December 2010.

All the cases were subjected detailed history and through clinical examination as per the structured pro forma. All the patients were subjected to skin biopsies (punch biopsy of $5 \mathrm{~mm}$ diameter) from the active part of the lesion after taking an informed consent.

Skin tissue was fixed in $10 \%$ formalin, serial sections of 5 micron thickness were stained with hematoxylin and eosin stain as well as by modified Fire Faraco for acid fast bacilli (in specified cases).
Histological examination of the skin tissue section was performed and classified according to Ridley Jopling classification. Cases were analysed in the pre structured proforma.

Statistical Analysis: Qualitative data were expressed as a frequency or percentage \& diagrammatic presentation was expressed using bar diagram. Correlation analysis was performed using Matthew's Correlation coefficient.

\section{RESULTS}

This prospective study out of the 42 cases the male female ratio was $1: 1$. According to the Age group range from 14 years to 75 years with maximum patients fall between 41 to 60 years (17 patients). (table 1)

Table No. 1: Demographic characteristics of Leprosy patients.

\begin{tabular}{|l|l|l|l|}
\hline Demographic variables & Frequency & Percentage \\
\hline \multirow{3}{*}{ Gender } & Male & 21 & $50.0 \%$ \\
\cline { 2 - 4 } & Female & 21 & $50.0 \%$ \\
\hline & $0-20$ & 7 & $16.6 \%$ \\
\cline { 2 - 4 } & $21-40$ & 8 & $19.0 \%$ \\
\cline { 2 - 4 } & $41-60$ & 17 & $40.0 \%$ \\
\cline { 2 - 4 } & $61-80$ & 10 & $23.8 \%$ \\
\hline
\end{tabular}

The majority of the cases were revealing of clinical leprosy spectrum in the Borderline Tuberculoid group was 16 cases followed by the Tuberculoid was 13 cases, 7 cases were Indeterminate Leprosy, Borderline lepromatous leprosy was 5 cases \& 1 was Lepromatous. (Table 2)

\section{- Clinical Examination Histopathological Examination}

22

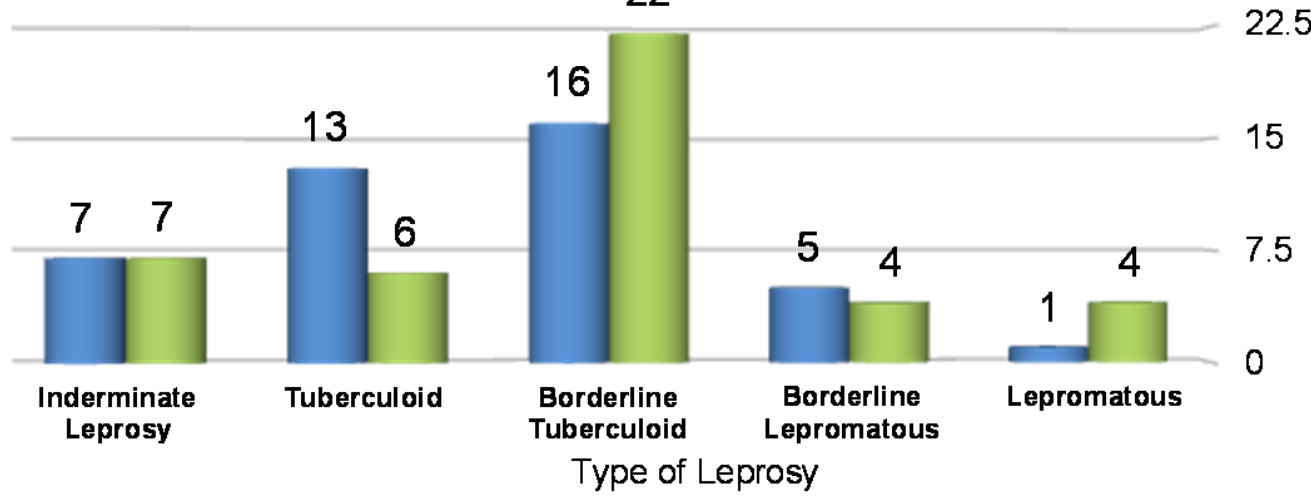

Figure No. 1: Clinico - Histopathological Findings 
Bhuvaneshwari Dewangan. Correlation of clinico-histopathological findings in leprosy patients at tertiary care hospital, Bhilai, $C G$.

Table No. 2: Distribution of clinical \& Histopathological leprosy spectrum in the study subjects.

\begin{tabular}{|l|l|l|}
\hline Type of Leprosy & No. Of cases in Clinical Examination & No. Of cases in Histopathological Examination \\
\hline Indeterminate Leprosy (IL) & $7(16.6 \%)$ & $7(16.6 \%)$ \\
\hline Tuberculoid (TT) & $13(30.9 \%)$ & $6(14.2 \%)$ \\
\hline Borderline Tuberculoid (BT) & $16(38.0 \%)$ & $22(52.3 \%)$ \\
\hline Borderline Lepromatous (BL) & $5(12.1 \%)$ & $4(9.5 \%)$ \\
\hline Lepromatous (LL) & $1(2.3 \%)$ & $4(9.5 \%)$ \\
\hline
\end{tabular}

Also Histopathological leprosy were observed in 41 cases. Out of these, maximum 22 cases were found in Borderline Tuberculoid followed by 7 cases were found in Indeterminate Leprosy, 6 cases were Tuberculoid, 4 cases were Borderline lepromatous \& 2 cases were found in Lepromatous. (Table 2)

Table No. 3: Correlation of Clinical and Histopathological findings.

\begin{tabular}{|l|l|l|}
\hline Sr. No. & Type of Leprosy & Correlation value \\
\hline 1 & Indeterminate Leprosy (IL) & 0.857 \\
\hline 2 & Tuberculoid (TT) & 0.461 \\
\hline 3 & Borderline Tuberculoid (BT) & 0.812 \\
\hline 4 & Borderline Lepromatous (BL) & 0.60 \\
\hline 5 & Lepromatous (LL) & 1.00 \\
\hline
\end{tabular}

Maximum clinico - histopathological correlation was seen in LL (1.000), followed by IL (0.857). The Overall clinic histopathological correlation was seen in 0.69. (table 3).

\section{DISCUSSION}

This was a protective study involving total 42 untreated clinically suspected cases of leprosy attending the dermatology and Venereology department of J. L. N. Hospital \& Research centre, Bhilai from March 2009 to December 2010.

This study shows that the male female ratio was 1:1. Although leprosy, males are affected more often as compared to females generally in the proportion of 2:1. Similarly Mc. Daugall \& Ponnighaus have reported a statistically significant preponderance of females in their study in Northern Malawi. ${ }^{(8)}$

This study found that the majority of the cases were belonging to the age group between 41 to 60 years \& the patients age ranged from 14 to 75 years. This observation is consistent with the age distribution of leprosy on prevalence data.
In the clinical spectrum majority 16 of the cases were found in the Borderline Tuberculoid \& 13 of the cases were found in the Tuberculoid. Also Histopathological features, majority 22 of the cases were found in the Borderline Tuberculoid.

The overall Clinico histopathological correlation of leprosy cases in our study was $69 \%$. Similar results were obtained by Jerath and Desai ${ }^{(9)}$, Bhatia et al. ${ }^{(10)} \&$ Kar et al. ${ }^{(11)}$ which was $68.5 \%$, $69 \%$ \& $70 \%$ respectively. The Overall statistical correlation i.e. kappa coefficient was found to be 0.64 , which denotes good strength of agreement between Clinical and Histopathological diagnosis.

\section{CONCLUSION}

The disparity between the clinical and histopathological observation was anticipated because the parameters used for the histopathological classification are well defined, precise and also take into account the immunologic response of the tissue, while clinical classification gives recognition only to the gross appearance of the lesions which is due to the underline pathological change. Moreover a sizeable proportion of cases of $\mathrm{BB}, \mathrm{BB}, \& \mathrm{BL}$ continuously change immunological spectrum and histological classification gives a better indication for any recent shift of a case position in the spectrum. In some early cases, clinical signs and symptoms may precede the presently known characteristic tissue changes or vice versa. If a biopsy is taken at an early stage, there is likely to be discordance between the clinical and histopathological observations. As false negative result may occur if biopsy is taken from non lesions site, biopsy from the lesion which is morphologically suggestive of clinical diagnosis, serial biopsies from the same lesion or from the paired lesions 
should be studied for better clinics histopathological correlation.

\section{REFERENCE}

1. Multi drug therapy against leprosy. Development and implementation over the past 25 yrs, WHO, Geneva 2004. (WHO/CDS/CEE/2004.46).

2. Frist T. Don't Treat me like I have Leprosy. London, International Federation of Anti Leprosy Association (ILEP), 1996.

3. WHO. Global Leprosy situation. Beginning of 2008, Wkly Epidemiol Rec, 2008; 83: $293-300$.

4. WHO. Leprosy Fact Sheet N 101, Feb. 2010, available at http://www.who.int/mediacentre/factsheets/f s101/en/

5. State-wise distribution of registered leprosy cases, DGHS, Govt. of India, nlep.nic.in/pdf/Annual Report 2007_08, Annexure-II.

6. WHO. Enhanced Global Strategy for further reducing the disease burden due to Leprosy (Plan period: 2011 - 2015), 2009; pp.28.
7. Joshi P. L. - National Scenario, NLEP and New paradigms. Kar H. K. And Bhushan Kumar, IAL Textbook of Leprosy First Edition, 2010; 35 - 36.

8. Mc Dougall A. C. Ponnighaus J. M., Fine P. E. M. Histopathological Examination of Skin Biopsies from an Epidemiological Study of Leprosy in Northern Malawi. Int. J. Leprosy. 1987; 55: 88 - 98.

9. Jerath VP, Desai SR. Diversities in Clinical and Histopathological classification of Leprosy. Lepr India. 1982; 54: 30.

10. Bhatia AS, Katoch K, Narayanan BR, Ramu G, Mukherjee A, Aavania RK. Clinical and Histopathological Correlation in the classification of leprosy. Int. J. Lepr 1993; 61: 433 - 438.

11. Kar PK, Arora PN. Clinico Histopathological study of macular lesions in leprosy. Indian J Lepr 1994; 66: 435 - 41.

How to cite this article: Dewangan B. Correlation of clinico-histopathological findings in leprosy patients at tertiary care hospital, Bhilai, CG. International Journal of Research and Review. 2021; 8(3): 28-31. 